\title{
Peculiarities of injection phenomena in heavily doped silicon structures and development of radiation-resistant diode temperature sensors
}

\author{
Yu.M. Shwarts, V.N. Sokolov, M.M. Shwarts, E.F. Venger \\ Institute of Semiconductor Physics, NAS Ukraine, 45 prospect Nauky, 03028 Kyiv, Ukraine \\ Phone: +38(044) 265 7463; e-mail: shwarts@isp.kiev.ua
}

\begin{abstract}
To explain the experimental behaviour of differential characteristics (ideality factor, differential resistance) before and after radiation influence, a theoretical model of injection current flow mechanisms for the silicon diode temperature sensors (DTSs) is proposed. The observed nonmonotonic dependencies of the ideality factor on the current are described well by the influence of generation-recombination and drift current components to the diffusion current of the minority carriers. The developed model allowed to find characteristic lifetimes of the minority carriers in the base and in the space-charge region of the diode structure with heavily doped base and emitter regions. Investigations of electrophysical and metrological characteristics of the DTSs allowed to reveal such operating regimes that are characterised by minimal influence of radiation on the device thermometric characteristics.
\end{abstract}

Keywords: radiation-resistance, diode temperature sensor, silicon diode structure.

Paper received 11.02.03; accepted for publication 16.06.03.

\section{Introduction}

The development of advanced semiconductor temperature sensors, in particular silicon ones, that are able to reliably operate under radiation environment above 1 Mrad is an actual task for extreme electronics [1]. Silicon diode temperature sensors (DTSs) are distinguished by a wide measured temperature range and high sensitivity, stability and reproducibility, high interchangeability [2]. However, widely represented on the market these DTSs continued not be suitable for applications under radiation conditions, as their thermometric characteristics underwent considerable temperature shift from $3 \mathrm{~K}$ to $18 \mathrm{~K}$ within actual for practical applications range of $77-350 \mathrm{~K}$ already at the dose of $10^{6} \mathrm{rad}$ [3].

The formation of thermometric characteristics of DTSs is determined by physical processes to occur in them. Therefore, the development of DTSs with predictable behaviour of their main characteristics under irradiation conditions and determination of optimal operation regimes are impossible without study of the current flow mechanisms in the device and their connection to constructive-technological parameters of semiconductor structures.
The present work is devoted to experimental and theoretical investigations of peculiarities of injection phenomena in heavily doped $p-n$ silicon structures under influence of irradiation with the purpose of development of radiation-resistant DTSs.

\section{Samples and experimental methods}

The DTSs investigated in this work have been developed by the industrial technology of manufacturing of diode chips on the base of heavily doped silicon structures. The concentration of boron in the test structures and in the base region of diodes is $\sim 2.10^{17} \mathrm{~cm}^{-3}$ and of phosphorous in the emitter region is $\sim 10^{20} \mathrm{~cm}^{-3}$.

The measurements of the conductivity, the Hall effect of test structures, the current-voltage characteristics (CVCs) of DTSs in the current interval of $10^{-11}-10^{-2} \mathrm{~A}$ have been carried out in the range of temperatures 77$400 \mathrm{~K}$ before and after irradiation. The silicon structures have been irradiated by $\gamma$-quantum $\mathrm{Co}^{60}$ in the set MRX- $\gamma-25 \mathrm{M}$ with the dose rate of gamma radiation of $200 \mathrm{rad} / \mathrm{s}$ at the temperature $320 \mathrm{~K}$.

The measurements of the temperature response curves (TRCs) have been carried out on the metrological stand 
Yu.M. Shwarts et al.: Peculiarities of injection phenomena in heavily ...

UGT-A in the range of excitation current from $0.1 \mu \mathrm{A}$ to $100 \mu \mathrm{A}$. An accuracy of the excitation current keeping was $\pm 0.05 \%$. A calibration accuracy of the DTSs is $0.03 \mathrm{~K}$.

\section{Experimental results}

From the experimental CVCs measured before and after irradiation, the ideality factor $m$ has been extracted by

$$
m=\left(q / k_{B} T\right)(d U / d \ln I),
$$

where $I$ is the direct current, $U$ is the voltage drop across the diode structure, $k_{B}$ is the Boltzmann constant, $q$ is the electron charge, $T$ is the absolute temperature.

In Fig. 1, we present the dependencies of the ideality factor $m$ on the current $I$ at the temperatures $220 \mathrm{~K}$ and $330 \mathrm{~K}$ for pre- and post-irradiation DTSs. The following peculiarities in the behaviour of the ideality factor can be pointed out:

a). All the dependencies $m(I)$ corresponding to the measurements before and after irradiation have a characteristic minimum. The value of the characteristic current in the minimum, $I_{\min }$, is increased with the irradiation dose $D$ from $I_{\min } \approx 10^{-6} \mathrm{~A}(D=0)$ to $I_{\min } \approx 10^{-5} \mathrm{~A}$ $\left(D=10^{7} \mathrm{rad}\right)$. The respective minimum value of the ideality factor $m_{\min }$ in the pre-irradiation dependence $m(I)$ is less than that corresponding to the post-irradiation one.

b). With increasing $I$, the dependencies $m(I)$ have approximately linear character. The slope of such the dependence is rather larger for the pre-irradiation measurements in comparison with the post-irradiation ones. The intersection point $C$ of these dependencies corresponds to the current $I \approx 10^{-4} \mathrm{~A}$. In the vicinity of the point $C$, the ideality factor and differential resistance are least influenced by the irradiation.

In Fig. 2, we show the temperature shift $\Delta T$ of the TRC due to the influence of irradiation for DTSs for the excitation current of $100 \mu \mathrm{A}$. As seen, the value of $\Delta T$

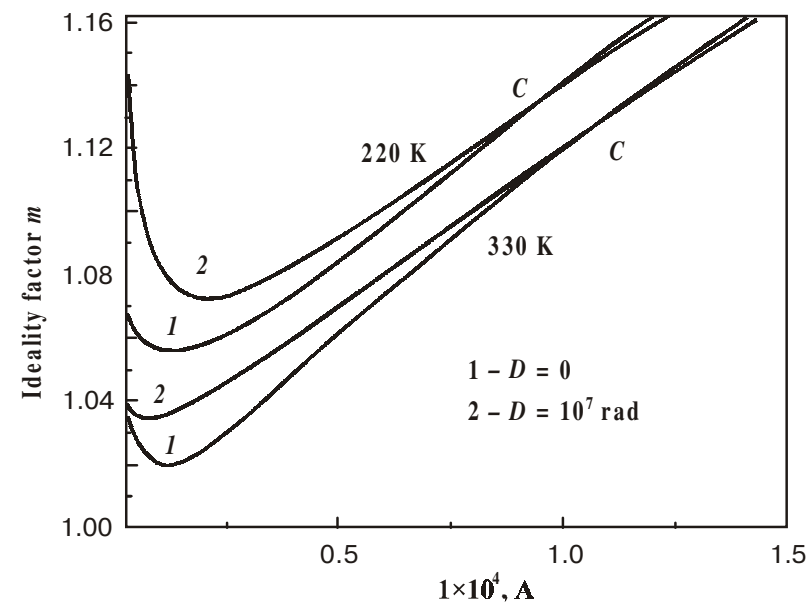

Fig. 1. Current dependencies of the ideality factor $m$ before and after gamma irradiation dose $D=10^{7} \mathrm{rad}$ at the temperatures of 220 and $330 \mathrm{~K}$.

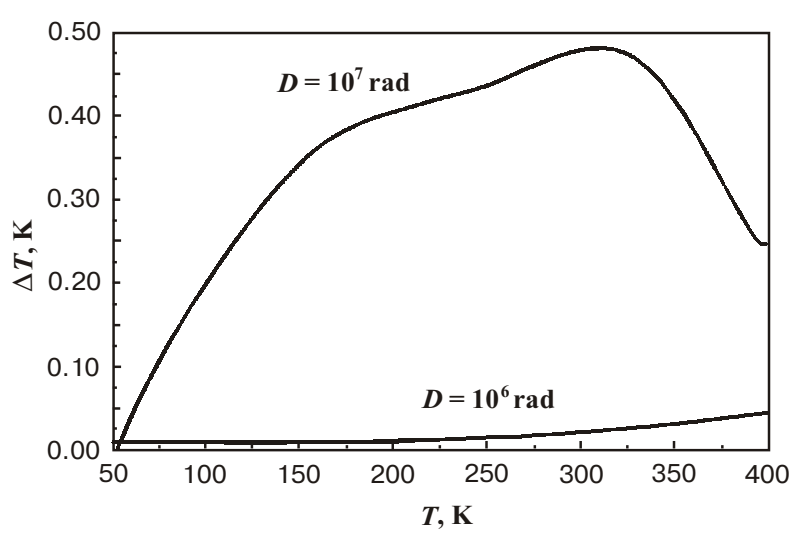

Fig. 2. Temperature shift $\Delta T$ as a function of temperature $T$ due to gamma irradiation doses from $\mathrm{Co}^{60}$ source. The excitation current is of $100 \mu \mathrm{A}$.

does not exceed $0.03 \mathrm{~K}$ for the dose $10^{6} \mathrm{rad}$ and $0.5 \mathrm{~K}$ for the dose $10^{7} \mathrm{rad}$.

In the next section we develop a simple theoretical model to explain the observed peculiarities of the ideality factor. On the base of this model such operating regimes of the DTSs are predicted that are least influenced by irradiation.

\section{Theory}

The established in the experiment proximity of $m(I)$ to unity testifies that the dominant carrier transport mechanism in the forward biased diode structure under study is the diffusion current of the minority carriers [4]

$I_{D}=I_{D S} \exp \left(q U_{0} / k_{B} T\right)$,

where $I_{D S}$ is the diffusion saturation current, $U_{0}$ is the voltage drop across the space-charge region of the junction.

The observed deviation of CVC from the ideal behaviour in the considered range of temperatures and currents may be explained with taking into account two different carrier transport mechanisms. The first one is the generation-recombination current [4], that can become essential with decreasing excitation current,

$I_{R}=I_{R S} \exp \left(q U_{0} / 2 k_{B} T\right)$,

where $I_{R S}$ is the respective saturation current. Another carrier transport mechanism that can become essential with increasing the excitation current is the current inherent in a pre-high injection regime [5]. These three current flow mechanisms must simultaneously be included in the theoretical model used for explanation of the experiment and analysis of radiation resistance of the DTSs.

For analysis of the CVCs in the pre-high injection regime we take into account redistribution of the applied voltage $U$ between the space-charge region of the junction $U_{0}$ and the diode base $U_{\nabla}$ due to the appearance of the gradient electric field $E_{\nabla}$ [5]. Respectively, new fea- 


\section{Yu.M. Shwarts et al.: Peculiarities of injection phenomena in heavily ...}

tures of the injection phenomenon in the diode structure should be considered, such as the drift component of the current due to the field $E_{\nabla}$; modification of the minority carrier distribution in the $p$-type base caused by the field $E_{\nabla}$ in comparison with that determined by the ideal diffusion carrier transport.

The minority carriers (electrons) injected into the diode base produce an excess negative charge that must be neutralised with an equal portion of a positive charge, as it is required by the charge neutrality condition: $n-n_{p 0}=$ $=p-p_{p 0}$. Here $n, p$ are the electron and hole concentrations in the $p$-type base and $n_{p 0,} p_{p 0}$ are their equilibrium values, respectively. Such regime is reached due to the majority carriers outgoing from the $p^{+}$-contact which nonuniform distribution in the base is kept with the electric field $E_{\nabla}$. At that, the diffusion and drift contributions to the majority carrier current density $j_{p}$ are approximately counterbalanced:

$j_{p}=q \mu_{p} p E-q D_{p} \frac{d p}{d x}=0$

where $\mu_{p}$ and $D_{p}$ are the hole mobility and diffusion coefficient. From this condition we obtain for the electric field $E_{\nabla}=\left(D_{p} / p \mu_{p}\right)(d p / d x)$ that results in a gradient voltage $U_{\nabla}$ across the base region

$U_{\nabla}=\int_{0}^{d} E_{\nabla}(x) d x=\frac{D_{p}}{\mu_{p}} \ln \frac{p(d)}{p(0)}$.

Here $p(0)$ and $p(d)$ are the hole concentrations at the space-charge region boundary on the base side $\left(x=x_{p}=\right.$ $=0)$ and at the $p^{+}$-contact $(x=d)$. Thereby, the voltage drop across the diode is

$U=U_{0}+U_{\nabla}$

Now the minority carrier current density $j_{n}$ includes, in contrast to the ideal case (2), both the drift and diffusion contributions

$$
j_{n}=q \mu_{n} n E+q D_{n} \frac{d n}{d x}
$$

where $\mu_{n}$ and $D_{n}$ are the electron mobility and diffusion coefficient. Using the Einstein relation $\mu_{n}=\left(q / k_{B} T\right) D_{n}$ and substituting for the electric field $E=E_{\nabla}$, the current density $j_{n}$ can be written as

$j_{n}=q D_{e f f}(n) \frac{d n}{d x}$.

Note that it can be interpreted in terms of the nonlinear diffusion transport of electrons with the effective diffusion coefficient $D_{\text {eff }}(n)$ dependent on the electron concentration

$D_{e f f}(n)=D_{n}\left(1+\frac{n}{n+p_{p 0}-n_{p 0}}\right)$.
The drift-diffusion current density through the $n^{+}-p$ junction can be written as

$j_{D D}=q D_{e f f}[n(0)]\left(\frac{d n}{d x}\right)_{x=0}$.

Below, we consider the case $n_{p 0}<<\Delta n<<p_{p 0}$. Then, the diffusion coefficient $D_{e f f}$ is reduced to

$D_{e f f}=D_{n}\left(1+\frac{\Delta n}{p_{p 0}}\right)$.

To obtain an expression for the CVC, we have found spatial distributions of the minority carrier concentration $n(x)$ in the base under the drift-diffusion transport and have calculated the derivative $(d n / d x)_{x=0}$ used in Eq.(9). Then using these results and the relation (6), the drift-diffusion current (9) can be put down as

$I_{D D}=I_{D S}[1-2 / 3 \alpha(U)] \exp \left(q U / k_{B} T\right)$,

where we have introduced the small parameter $\alpha(U) \equiv$ $\equiv\left(n_{i}^{2} / p_{p 0}^{2}\right) \exp \left(q U / k_{B} T\right)<<1$.

The total current through the diode can be obtained by combining the drift-diffusion current (11) and the generation-recombination current (3):

$$
\begin{aligned}
& I=I_{D S}\left[1-\frac{2}{3} \alpha(U)\right] \exp \left(q U / k_{B} T\right)+ \\
& +I_{R S}\left[1-\frac{1}{3} \alpha(U)\right] \exp \left(q U / 2 k_{B} T\right) .
\end{aligned}
$$

Then, using Eqs.(1) and (12), we arrive to explicit expression for the ideality factor $m$ as a function of the excitation current

$m(I)=1+\frac{I}{I_{1}}+\sqrt{\frac{I_{2}}{I}}$.

Here, the characteristic currents $I_{1,2}$ are given by

$I_{1}=\frac{3 p_{p 0}^{2} I_{D S}}{2 n_{i}^{2}}, \quad I_{2}=\frac{I_{R S}^{2}}{4 I_{D S}}$.

The sum on the right-hand side of Eq.(13) is related to the ideal diffusion current, the pre-high injection current (the second term), and the generation-recombination current (the third term). The asymptotic behaviour of the ideality factor with increasing current is given by a linear dependence $m(I) \cong 1+\left(I / I_{1}\right)$ with the characteristic slope

$\operatorname{tg} \varphi \propto 1 / I_{1}$.

With decreasing current, an approximate dependence $m(I) \cong 1+\left(I_{2} / I\right)^{1 / 2}$ can be obtained. For $I \cong\left(I_{1}^{2} I_{2}\right)^{1 / 3}$, both the last terms in (13) are of the same order of magni- 
tude that leads to a minimum in the $m(I)$-dependence. It is easy to verify that at the minimum the ideality factor is

$m_{\min }=1+\frac{1}{2}\left(\frac{3 n_{i} I_{R S}}{p_{p 0} I_{D S}}\right)^{2 / 3}$,

with $I_{\min }$ being

$I_{\min }=I_{D S}\left(\frac{3 p_{p 0}^{2} I_{R S}}{8 n_{i}^{2} I_{D S}}\right)^{2 / 3}$.

Thus, taking into account the above discussed three carrier transport mechanisms in the diode structure has allowed us to describe the observed nonmonotonic dependencies of the ideality factor on the excitation current.

\section{Discussion}

From the measurements of Hall effect on the test structures we have found that the carrier mobility within the investigated temperature range is least sensitive to the influence of gamma irradiation. The concentration of induced radiation defects does not exceed the value of $\sim 10^{15} \mathrm{~cm}^{-3}$ for the doze $D=10^{7} \mathrm{rad}$. Because of a high level doping of the diode base, the concentration of induced radiation defects appears to be insufficient for appreciable influence to the carrier density and the basic carrier scattering mechanisms, which are responsible for formation of the carrier mobility in the considered temperature range. Therefore, in the diode structures under investigation within the limits of radiation doses used, the resistivity $\rho$ is not a critical parameter to considerably affect the radiation resistance of the DTSs. Thus, we should consider the minority carrier lifetime $\tau$ as the basic critical parameter of radiation resistance of our diode structures.

We propose a new method to determine the carrier lifetime in the base $\tau_{n}$ and in the space-charge region $\tau_{r}$ from the investigated dependencies of $m(I)$. By substituting the explicit expressions [4] for the saturation currents $I_{D S}=\left[q S n_{i}{ }^{2} / N_{A}\right.$ th $\left.\left(d / L_{n}\right)\right]\left(D_{n} / \tau_{n}\right)^{1 / 2}$ and $I_{R S}=$ $=q S W n_{i} / 2 \tau_{r}$ in (16) and (17), the lifetimes $\tau_{n}$ and $\tau_{r}$ can be expressed as

$$
\begin{aligned}
& \tau_{n}=\left(\frac{3}{2} q S p_{p 0} \sqrt{D_{n}} \operatorname{tg} \varphi\right)^{2}, \\
& \tau_{r}=\frac{3 W \sqrt{\tau_{n}}}{4 \sqrt{2 D_{n}}\left(\delta m_{\min }\right)^{3 / 2}},
\end{aligned}
$$

where $S$ is the effective junction area, $W$ is the spacecharge region width, $\delta m_{\min }=m_{\min }-1$. Using the experimental values for $d m_{\min }$ and $\operatorname{tg} \varphi$ and electrophysical and design parameters for the DTSs, the lifetimes $\tau_{n}$ and $\tau_{r}$ have been calculated for the pre- and post-irradiation measurements in the investigated temperature range. The results are presented in Fig. 3. The obtained values for the pre-irradiation lifetime $\tau_{n}{ }^{0}$ agree well with available literature data on the minority carrier lifetime in the heavily doped silicon diffused layers [6].

The irradiation reduces the minority carrier lifetime $\tau_{n}$ in the base only slightly, while the lifetime in the spacecharge region $\tau_{r}{ }^{D}$ after irradiation is less approximately in an order of magnitude in comparison with that $\tau_{r}^{0}$ before irradiation. As in regime of pre-high injection the ideality factor (13) is determined mainly by the dependence $m(I) \cong 1+I / I_{1}$, then according to definition of $I_{1}$ and $I_{D S}$, the characteristic slope of $m(I)(15)$ changes with variation of the lifetime $\tau_{n}$ as $\operatorname{tg} \varphi \sim\left(\tau_{n}\right)^{1 / 2}$. Therefore, the slope after irradiation becomes lower than that before irradiation. On the other hand, according to (16) and definitions of $I_{D S}$ and $I_{R S}$, the ideality factor at its minimum $m_{\min }$ varies as $\sim\left(\tau_{n} / \tau_{r}^{2}\right)^{1 / 3}$. Such combination of the lifetimes results in an increase of the value of $m_{\min }$ after irradiation. Therefore, the resulting combination of both changes in the slope and the ideality factor minimum after irradiation leads to the presence of the cross point $C$ of the curves $m(I)$ for the pre- and post irradiation measurements (Fig. 1).

It is important to note that for operating regimes corresponding to a vicinity of the point $C$ the ideality factor is least sensitive to influence of irradiation. It indicates that the dominant current flow mechanism in the DTSs has not undergone an essential modification. For the dominant diffusion carrier transport mechanism, the influence of radiation on the TRC (in the temperature equivalent $\Delta T$ ) is determined by the influence on the saturation current $I_{D S}$, that is, on the lifetime, mobility, and concentration of carriers in the base. As was mentioned above, for the developed DTSs the changes in these three electrophysical parameters under irradiation are not considerable. At the same time, the observable more significant change in the lifetime $\tau_{r}$ in the space charge region of the diode cannot result in a noticeable modification of

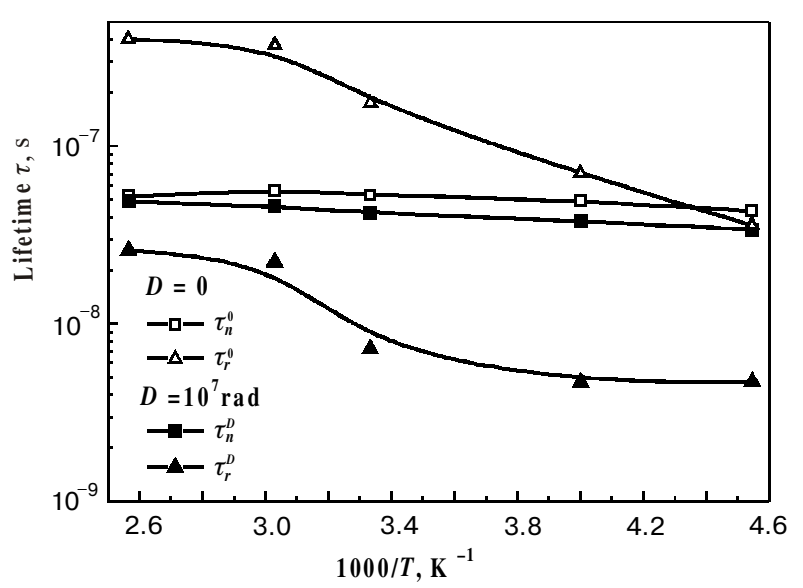

Fig. 3. Electron minority carrier lifetimes $\tau_{r}$ and $\tau_{n}$ calculated from the current dependencies of the ideality factor before and after irradiation at different temperatures.

SQO, 6(2), 2003 


\section{Yu.M. Shwarts et al.: Peculiarities of injection phenomena in heavily ...}

the TRC as well, since the contribution of the generation-recombination current to the total current through the diode is small. This is the basic reason of radiation resistance of the developed DTSs reached in the investigated temperature range and the excitation current regime.

\section{Conclusions}

The developed theoretical analytical model taking into account peculiarities of injection current flow mechanisms for the DTSs explains well the experimental behaviour of differential characteristics (ideality factor, differential resistance) under radiation influence. The nonmonotonic current dependencies of the ideality factor are adequately described by the influence of the generation-recombination and drift current components in combination with the diffusion current of the minority carriers. The position of the minimum in the dependencies of $m(I)$ is determined by the competition of these two additional currents. The lifetimes of the minority carriers have been found separately in the base and in the space-charge region of the diode structure. The analysis of current flow mechanisms in the DTSs has allowed to reveal optimal op- erating regimes for the sensor which are characterised high radiation resistance. Such the regimes provide the temperature shift $\Delta T$ caused by gamma irradiation from $\mathrm{Co}^{60}$ with the doze up to $10^{6}$ which does not exceed of $0.03 \mathrm{~K}$. With increasing doze of the irradiation up to $10^{7} \mathrm{rad}$, the value of $\Delta T$ does not exceed of $0.5 \mathrm{~K}$ that completely meets the requirements to temperature sensors for practically important cases.

\section{References}

1. Conference on Electronics for Extreme Environments, 1999NASA/JPL, Pasadena Convention Center, Pasadena, 1999, http://extelect.jpl.nasa.gov/conference/.

2. L.G. Rubin, Cryogenic thermometry: a review of progress since 1982 // Cryogenics 37, pp. 341-356 (1997).

3. J.M. Swarts, Temperature measurement and control, Lake Shore Product Catalog available from Lake Shore Cryotronics, Inc., Westerville, OH, 43082, USA, (1999).

4. S.M. Sze, Physics of Semiconductor Devises, $2^{\text {nd }}$ ed., Wiley, New York, (1981).

5. I.V. Vikulin, V.I. Stafeev, Fizika poluprovodnikovyh priborov, $2^{\text {nd }}$ ed., Radio i svyaz", Moscow, (1990).

6. D.J.Roulston, N.D. Arora, S.G. Chamberlain, Modelling and measurement of minority-carrier lifetime versus doping in diffused layers of $\mathrm{n}^{+}-\mathrm{p}$ silicon diodes // IEEE Trans. Electron Devices ED-29, pp.284-291 (1982). 